\title{
Energetics of Electron-Transfer Reactions in Soft Condensed Media
}

\author{
DMITRY V. MATYUSHOV \\ Department of Chemistry and Biochemistry, Department of \\ Physics and Center for Biological Physics, Arizona State \\ University, Tempe, Arizona 85287-1604
}

Received September 28, 2006

\section{ABSTRACT}

The coupling of electronic transitions within molecules to condensed-phase media involves a complex hierarchy of spatial and dynamical scales. Thermodynamics of activation is related to the length scale of microscopic interactions reflected in the nonArrhenius reaction kinetics. Solvent dynamics make a particularly strong impact on the activation barrier when the time scale of the reaction is comparable to the relaxation time of the solvent, and the reaction barrier becomes nonergodic. Finally, molecular polarizability is responsible for complex nonparabolic free energy surfaces for electron transfer. We discuss the application of these ideas to soft condensed solvents such as supercooled liquids, liquid crystals, and photosynthetic reaction centers.

\section{Introduction}

Perhaps no other branch of chemical kinetics has contributed more to the fundamental understanding of the factors controlling activation barriers of reactions in liquids than the field of electron-transfer reactions (see reviews in refs 1-4). Recent advances in spectroscopic techniques examining the dynamics and thermodynamics of solute-solvent interactions, along with the progress in computer simulations, have led to new insights into the microscopic mechanisms and solvent modes driving activated transitions. In parallel to these developments, the list of solvents used for electron-transfer reactions quickly broadens to include complex soft condensed media such as liquid crystals, ${ }^{5}$ proteins, ${ }^{6}$ and structural glass formers. ${ }^{7}$ The combination of fast reactions with solvents characterized by either slow relaxation, anisotropy (existence of preferential directions), or inhomogeneity (existence of interfaces) has posed new challenges for the theory, at the same time opening new avenues for controlling chemical reactivity. This Account reviews these currently emerging directions along with outlooks into future studies of electronic transitions in condensed media.

The amount of Gibbs free energy, $\Delta G_{0}$, released or consumed in the transformation from reactants to products (reaction free energy) dramatically affects the magnitude of the reaction rate. The advent of the MarcusHush theory of electron transfer in the $1950 \mathrm{~s}^{8,9}$ has challenged the traditional understanding of the connec-

Dmitry V. Matyushov was born in Lvov, Ukraine, in 1963. He received his M.Sc. from the Moscow Institute for Physics and Technology and his Ph.D. from Kiev's State University. His research interests are in Chemical Physics and include electron-transfer processes, solvation, and theories of chemical activation.
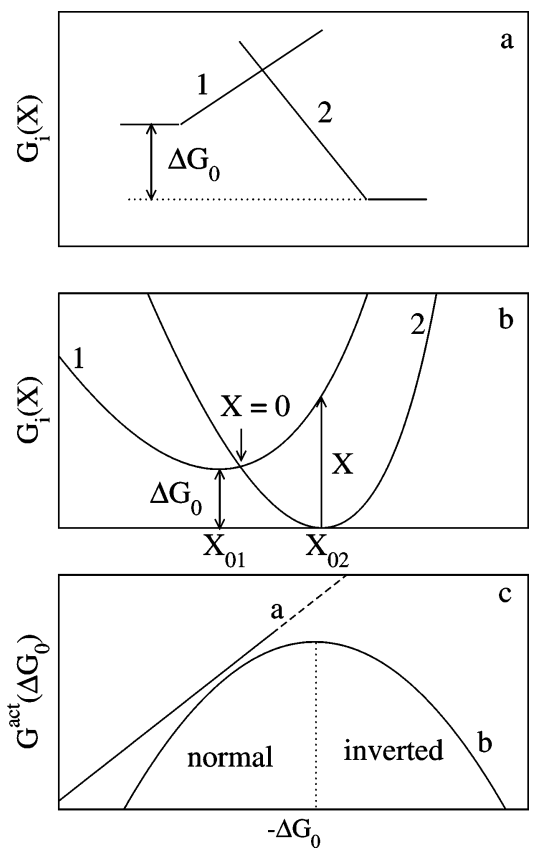

FIGURE 1. Linear (a) and nonlinear (generally nonparabolic when calculated from eqs 2 and 17, b) free energy surfaces and the dependence of the rate constant on the reaction free energy (energy-gap law, $c$ ). In panel $b$, the vertical arrow shows a vertical transition in which the energy changes by the energy gap $X$ (eq 2) but the entropy does not change. In panel $c$, the energy gap law is sketched for linear (marked as "a") and nonlinear (marked as "b") free-energy surfaces. The nonlinear energy gap law is quadratic close to its maximum and is linear in its exothermal wing.

tion between the reaction thermodynamics and reaction rates embodied into linear free energy relationships ${ }^{10}$ assuming the rate constant (in the log scale) to be a linear function of $\Delta G_{0}$. This linear dependence on $\Delta G_{0}$ is visualized in terms of free energy functions linear in some reaction coordinate. They cross to form the activation barrier, which, in turn, becomes linear in $\Delta G_{0}$ (Figure 1a). In contrast, in the Marcus-Hush theory, the free energy surfaces are parabolas (Figure 1b) and the activation barrier is bilinear (linear plus quadratic) in $\Delta G_{0}$. The rate constant first increases with increasing $-\Delta G_{0}$ (normal region) and then drops after passing the activationless transition at the top of the bell-shaped curve (inverted region, Figure 1c).

In both the linear and parabolic paradigms, one needs to specify some parameters in addition to $\Delta G_{0}$ in order to connect the activation barrier to the reaction Gibbs energy. For linear surfaces, these are the slopes of reactant and products lines, while for parabolas, these are curvatures, which are normally defined in terms of reorganization energies $\lambda_{1}$ and $\lambda_{2}$ for the initial (reactants, $i=1$ ) and final (products, $i=2$ ) states:

$$
\frac{1}{2 \lambda_{i}}=\left.\frac{\mathrm{d}^{2} G_{i}(X)}{\mathrm{d} X^{2}}\right|_{X=X_{0 i}}
$$

Here, the second derivative is taken at the parabola's minimum, $X_{0 i}$.

10.1021/ar7000167 CCC: $\$ 37.00$ @ 2007 American Chemical Society Published on Web 03/17/2007 
The single transition point at the intersection of reactant and product free energy surfaces hides the whole world of quantum tunneling, which can only occur at the resonance of the donor and acceptor electronic states. Since it is this diffusive climbing of the system to the point of resonance that is most relevant for the reaction, spectroscopic reaction coordinate $X=\Delta E$ (see below), equal to the instantaneous energy gap, $\Delta E=E_{2}-E_{1}$, between the electronic energies of the donor and acceptor, provides the most straightforward formulation of the problem. This collective coordinate, effectively incorporating all nuclear modes of the liquid affecting the energy gap, is clearly distinct from a commonly small number of actual nuclear coordinates used in describing reactions in the gas phase. The language of collective modes and excitations, so prevailant in solid-state physics and much less common in chemistry, is therefore actively used in theories of radiationless transitions in condensed media and for electron-transfer reactions in particular.

Once the donor-acceptor energy gap is chosen for the reaction coordinate, the requirement of conservation of energy imposes an important constraint on the shapes of the free energy surfaces, $G_{i}(X)$. The system entropy does not change in a vertical (photoinduced) transition at a given nuclear configuration (shown by the vertical arrow in Figure 1b), and the vertical gap between the free energy surfaces should be equal to the energy reaction coordinate, $X: 11,12$

$$
G_{2}(X)=G_{1}(X)+X
$$

Any pair of free energy surfaces used to model the energetics of electron-transfer reactions should comply with eq 2.

The main challenge in modeling the energetics of electron-transfer reactions and, more generally, chargetransfer transitions in molecular systems is how to relate the parameters used to build the free energy surfaces to observables available through laboratory or computer experiment. Spectroscopy, in principle, provides the complete solution to the problem since spectral intensity is proportional to the equilibrium distribution of the donoracceptor energy gaps. In practice, however, the activation barrier at $X=0$ (electronic resonance) is never accessible since it corresponds to a photon of zero energy. In fact, intensities only in close proximity of equilibrium points $X_{0 i}$, corresponding to maxima of spectral lines, can be measured. They are often available, along with curvatures $\lambda_{i}$, which can be extracted from widths of vibronic lines. These four spectroscopic parameters normally need to be reduced to three since the vertical transition energies include usually ill-known gas-phase components. They cancel out in the difference of excitation energies (Stokes shift). The experimentally measured Stokes shift still contains a component from intramolecular vibrations, which should be subtracted to obtain the solvent-induced Stokes shift $\hbar \Delta \omega_{\mathrm{st}}=X_{02}-X_{01} \cdot{ }^{13}$ To summarize, even for the best-studied systems, three or probably four param- eters, defining the positions of the minima and equilibrium curvatures of $G_{i}(X)$, can be obtained from spectroscopy.

The spectroscopic database is consistent with the requirement of thermodynamics that the two-state electron-transfer problem is, in principle, characterized by three thermodynamic parameters: $\Delta G_{0}$ and two, generally different, reorganization energies $\lambda_{1}$ and $\lambda_{2}$. The reorganization energies, defined in terms of free energy curvatures (eq 1), can be connected to small fluctuations of the system around equilibrium, which are still described by equilibrium statistical mechanics. Any extension of the model beyond the thermodynamic three-parameter input requires a nonequilibrium description.

The Marcus-Hush theory reduces the number of thermodynamic parameters to two: the free energy gap, $\Delta G_{0}$, and just one reorganization energy, $\lambda_{1}=\lambda_{2}$. This approximation allows one to use equal-curvature parabolas, which comply with the energy conservation requirement in eq 2. Even though this model has received enormous support over about 50 years of its application, ${ }^{1-4}$ the calculation of electron-transfer reorganization energies from microscopic interaction potentials is still in its infancy. ${ }^{14}$ We review the development of micrscopic models focusing primarily on general results, in particular on the splitting of the activation free energy into the entropy and enthalpy of activation. ${ }^{15,16}$ This will be followed by the discussion of the effects of freezing of nuclear degrees of freedom on the activation barrier resulting in nonergodic kinetics. Finally, we discuss the extension of the model into the three-parameter space allowing nonparabolic free energy surfaces.

\section{Microscopic Reorganization}

The donor-acceptor electronic energy gap is composed of a gas-phase component and the difference in the solute-solvent interaction potentials in the acceptor and donor states, $\Delta U_{0 s}$. The activated passage from the initial reactant state (e.g., $X_{01}$ in Figure $1 b$ ) to the activated state with the resonance of the donor and acceptor electronic levels $(X=0$ in Figure $1 b)$ is driven by thermal fluctuatons of the nuclear degrees of freedom of the solvent. The variance of the energy gap is then fully determined by the statistics of solute-solvent interactions, and one gets

$$
\sigma^{2}=\left\langle(\delta \Delta E)^{2}\right\rangle=\left\langle\left(\delta \Delta U_{0 s}\right)^{2}\right\rangle
$$

where the angular brackets stand for an ensemble average.

Since many molecular motions are coupled to the transferred electron, the overall distribution of the energygap fluctuations, according to the central limit theorem, is a Gaussian function

$$
P_{i}(X) \propto \exp \left[-\frac{\left(X-X_{0 i}\right)^{2}}{2 \sigma^{2}}\right]
$$

The solute-solvent interaction is normally given by a sum of all pairwise interactions of the solute with the solvent molecules. When such a pairwise decomposable potential is substituted into eq 3 , the variance, $\sigma^{2}$, splits into the 
single particle term, $\sigma_{\mathrm{I}}^{2}$, and two-particle term, $\sigma_{\mathrm{II}}^{2}::^{25}$

$$
\sigma^{2}=\sigma_{\mathrm{I}}^{2}-\sigma_{\mathrm{II}}^{2}
$$

The former represents fluctuations of a single particle of the solvent, uncorrelated from the rest of the solvent, while the latter refers to statistically averaged correlated fluctuations of two particles of the solvent. The second term physically represents the force acting on a given solvent molecule from the rest of the solvent, which tends to damp single-particle motions in response to the field of the solute. The solvent thus reacts to reduce the action of the solute external perturbation (Le Chatelier's principle).

Electronic transitions in polar liquids are mainly driven by thermal motions of solvent dipoles. ${ }^{1,4}$ Fluctuations of orientations of dipoles in polar liquids are highly correlated. Therefore, any change in orientation of a given solvent molecule, caused by the electric field of the solute, is strongly damped by the field of the surrounding dipoles. The consequence is close magnitudes of $\sigma_{\mathrm{I}}^{2}$ and $\sigma_{\mathrm{II}}^{2}$ components almost compensating each other in the variance $\sigma^{2}{ }^{15}$ This cancellation of single-particle and twoparticle contributions to the solvent response is a mathematical signature of the quasi-macroscopic nature of dipolar polarization. Such macroscopic response is described by the fluctuation-dissipation theorem (classical limit) ${ }^{17}$

$$
\sigma^{2}=2 k_{\mathrm{B}} T \lambda_{\mathrm{s}}, \quad \lambda_{\mathrm{s}}=\int_{0}^{\infty} \chi^{\prime \prime}(\omega)(\mathrm{d} \omega /(\pi \omega))
$$

where subscript "s" stands for the solvent and equality of two reorganization energies, $\lambda_{\mathrm{s}}=\lambda_{1}=\lambda_{2}$, according to the Marcus-Hush picture, is assumed in this and the next sections.

The use of the term "dissipation" is connected to the fact that the energy absorbed by, for instance, photoexciting the solute dissipates as heat with the rate proportional to $\omega \chi^{\prime \prime}(\omega)$, where $\chi^{\prime \prime}(\omega)$ in eq 6 is the imaginary part of the Stokes shift susceptibility. This function, $\chi^{\prime \prime}-$ $(\omega)=\pi \omega C(\omega) /\left(k_{\mathrm{B}} T\right)$, is experimentally accessible (from time-resolved emission spectroscopy ${ }^{13}$ ) as Fourier transform of the Stokes shift correlation function $C(\omega)$, where $C(t)=\langle\delta X(t) \delta X(0)\rangle$ is the time autocorrelation function of the fluctuations of the reaction coordinate $\delta X(t)=X(t)-$ $\langle X\rangle$.

The energy gap variance $\sigma^{2}$ reflects two distinct physical phenomena: thermal fluctuations around two equilibrium points $X_{0 i}$ (proportionality to $T$ ) and the finite amplitude of such fluctuations (proportionality to the inverse curvature $2 \lambda_{\mathrm{s}}$, see eqs 1 and 6 ). If $\lambda_{\mathrm{s}}$ is temperatureindependent, eq 6 predicts the Gaussian width $\sigma^{2}$ to be a linear function of $T$ crossing zero at $T=0$. In this case, the probability of reaching the activated state $X=0$ (Figure 1b) describes the Arrhenius kinetics

$$
P(0) \propto \mathrm{e}^{\left.-G^{\mathrm{act} /} / k_{\mathrm{B}} T\right)}
$$

where the activation free energy $G^{\text {act }}$ can be recast in the form of the celebrated Marcus equation ${ }^{1}$

$$
G^{\mathrm{act}}=\left(\Delta G_{0}+\lambda_{\mathrm{s}}\right)^{2} /\left(4 \lambda_{\mathrm{s}}\right)
$$

The present discussion is limited to classical nuclear modes; for the discussion of quatum effects, see ref 3 .

The microscopic structure of the polar liquid turned out to be responsible for a pronounced $T$-dependence of $\lambda_{\mathrm{s}}{ }^{18}$ (for reviews of experimental data see refs 16 and 19), which can be visualized by plotting $\sigma^{2}(T)$ against $T$. In contrast to the prediction of eq 6 with $\lambda_{\mathrm{s}}=$ const, $\sigma^{2}(T)$ crosses the vertical axes at a positive value (Figure 2). This positive intercept is related to a positive value of the reorganization entropy, which can be measured either at constant volume (subscript " $V$ ") or at constant pressure (subscript “ $P$ ”)

$$
S_{P, V}=-\left(\partial \lambda_{\mathrm{s}} / \partial T\right)_{P, V}
$$

Which entropy, $S_{P}$ or $S_{V}$, is larger is controlled by the sign of the isobaric expansivity of the solvent: $S_{P}>S_{V}$ at positive expansivity (as in Figure 2) and $S_{P}<S_{V}$ at negative expansivity. ${ }^{18}$

The quasi-macroscopic character of the dipolar solvent response is caused by the long range of electrostatic solute-solvent and dipolar solvent-solvent interactions. When either of the two is more short-ranged, the cancellation between single-particle and two-particle parts of the response is not perfect and the solvent response bears more microscopic features. The first signature of this change is that $\sigma^{2}$ in eq 6 does not scale linearly with temperature any more. For instance, the two-particle component, $\sigma_{\mathrm{II}}^{2}$, is just a small fraction of $\sigma_{\mathrm{I}}^{2}$ (see eq 5) for quadrupolar solvents, ${ }^{20}$ and this result holds true for all interactions that are more short-ranged than the dipole-dipole potential (e.g., induction and dispersion forces ${ }^{21}$ ). This fact has a profound effect on the temperature dependence of the energy gap variance. The variance of the gap, $\sigma_{\mathrm{sr}}{ }^{2}$, arising from all short-ranged interactions (subscript "sr") does not have an explicit temperature factor and depends on temperature mostly through the solvent density. Therefore, the temperature dependence of the variance at isochoric conditions can be given by the relation

$$
\sigma^{2}=2 k_{\mathrm{B}} T \lambda_{\mathrm{s}}+\sigma_{\mathrm{sr}}^{2}
$$

where $\sigma_{\mathrm{sr}}^{2}$ is essentially temperature-independent. When eq 10 is substituted into the probability of reaching the activated state at $X=0$ in eq 4 , the kinetics of electron transfer becomes distinctly non-Arrhenius, that is, the energy of activation (slope of the Arrhenius plot) in eq 7 depends temperature. The temperature dependence of the activation barrier becomes even more pronounced when the reaction loses ergodicity, as we discuss next.

\section{Nonergodic Reactions}

The description of the electron-transfer rates in terms of the equilibrium free energy of activation is based on transition-state ideas, which assume that equilibrium Boltzmann distribution along a reaction coordinate is 


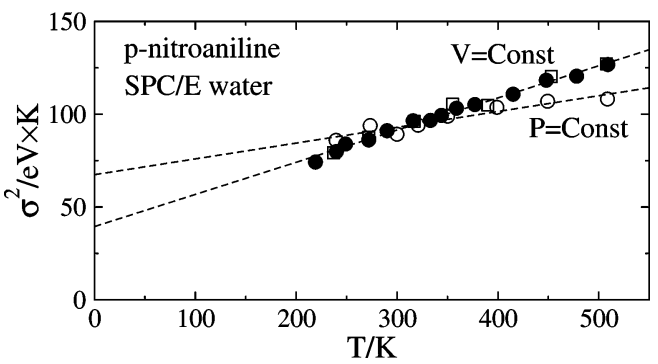

FIGURE 2. Gaussian width, $\sigma^{2}=2 k_{\mathrm{B}} T \lambda_{\mathrm{s}}$, of $p$-nitroaniline in SPC/E water obtained from molecular dynamics simulations ${ }^{18}$ at constant volume $(\boldsymbol{\bullet}, \square)$ and constant pressure $(O)$. Circles indicate the charge-separation reaction associated with optical absorption, whereas squares refer to the charge-recombination reaction associated with optical emission. The dashed lines are linear regressions through the simulation points.

maintained for the reactant states up to the transitionstate surface..$^{22}$ Possible limitations of this picture are well recognized. When the time scale of passage of the barrier top is comparable to the relaxation time of the medium, the population of the activated state becomes depleted, and one arrives at the Kramers kinetics in which the rate pre-exponent is affected by the solvent friction (viscosity). ${ }^{22}$ For electron-transfer reactions, this regime corresponds to solvent-controlled reactions when the reaction pre-exponent includes some characteristic time of polarization relaxation. This dynamic solvent effect has indeed been observed in some systems, ${ }^{23}$ although it has been recognized in recent years that the range of relaxation times at which it can be observed is relatively narrow due to the competition of the diffusional dielectric relaxation with classical ${ }^{24}$ and quantum ${ }^{25}$ solute vibrations and ballistic solvent dynamics. ${ }^{26}$

Essentially for all reactions affected by the solvent, one may anticipate the next step in the hierarchy of relaxation times when the solvent relaxation becomes even slower. Equilibrium solvation energy is formed by the system visiting all parts of its phase space on the infinite (on the molecular scale) observation time of the equilibrium thermodynamics. Once the time scale of the reaction, that is, the overall time required to climb the activation barrier from the reactant bottom to the top, becomes comparable to the relaxation time of a given nuclear mode driving activation, the system looses ergodicity. ${ }^{27}$ Physically it means that not all possible configurations of the solvent can be realized on the time scale of the reaction and the magnitude of the nuclear solvation energy decreases. ${ }^{24,28}$

The transition to nonergodic behavior can be observed in simulations ${ }^{29}$ by lowering the solvent temperature, which has the effect of increasing the dielectric relaxation time of a polar solvent. Once this slow relaxation time crosses the length of simulated trajectory, the reorganization energy turns from its thermodynamically predicted rise $\left(S_{P, V}>0\right)$ and starts decreasing with lowering temperature (Figure 3). The temperature, $T^{*}$, of this turnover marks the onset of nonergodic solvent fluctuations. After the initial decay at $T<T^{*}$, the reorganization energy levels off at the value $\lambda_{\mathrm{G}}$ corresponding to fast ballistic motions

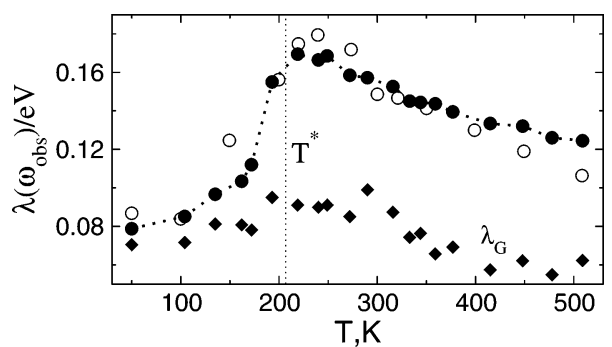

FIGURE 3. Solvent reorganization energy of $p$-nitroaniline in SPC/E water obtained from molecular dynamics simulations ${ }^{29}$ at constant volume (O) and constant pressure (O). The reorganization energy is ergodic at $T>T^{*}$. The component of reorganization energy corresponding to reorientation of permanent dipoles loses ergodicity below $T^{*}$ when the reorganization energy drops to the value $\lambda_{\mathrm{G}}$ arising from fast ballistic solvent modes characterized by the Gaussian component in the Stokes shift time correlation function ${ }^{30}$ (>).

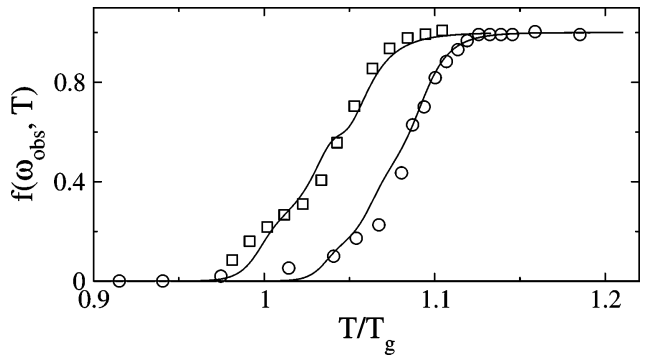

FIGURE 4. Nonergodicity function obtained as the ratio of nonergodic and equilibrium reorganization energies for quinoxaline chromophore dissolved in supercooled 2-methylhydrofuran $\left(T_{\mathrm{g}}=\right.$ $91 \mathrm{~K}, \square)$ and butyronitrile $\left(T_{\mathrm{g}}=97 \mathrm{~K}, \bigcirc\right)$. The points are experimental data $^{31,32}$ and the lines are theoretical calculations. ${ }^{29,32}$

in the liquid, ${ }^{30}$ which require much shorter observation times to become dynamically arrested.

The change of the nuclear solvation energy at the transition to nonergodicity can be described analytically by noting that the solvent modes with relaxation frequencies below the observation frequency $\omega_{\mathrm{obs}}=\tau_{\mathrm{obs}}{ }^{-1}$ do not contribute to the overall frequency-integrated response of the solvent. The nonergodic reorganization energy can be obtained by applying a stepwise frequency filter, $\omega>$ $\omega_{\text {obs }}$, to eq $6^{27,29}$

$$
\lambda\left(\omega_{\mathrm{obs}}\right)=\int_{\omega_{\mathrm{obs}}}^{\infty} \chi^{\prime \prime}(\omega)(\mathrm{d} \omega /(\pi \omega))
$$

A fixed observation time $\tau_{\text {obs }}$ is realized in the spectroscopic experiment where $\tau_{\mathrm{obs}}$ is either given by the lifetime of the emitting state of a chromophore or determined by the apparatus. Equation 11 allows one to calculate the temperature dependence of the nonergodic Stokes shift based on the equilibrium ergodic Stokes shift susceptibility, $\chi(\omega)$ (see the discussion after eq 6). The performance of the theory applied to normalized Stokes shift of quinoxaline phosphorescent dye in supercooled 2-methylhydrofurane and butyronitrile ${ }^{31,32}$ is quite satisfactory (Figure 4). Theoretical calculations are based on the formalism for $\chi(\omega),{ }^{33}$ experimentally measured dielectric constants as functions of frequency, and quantum- 


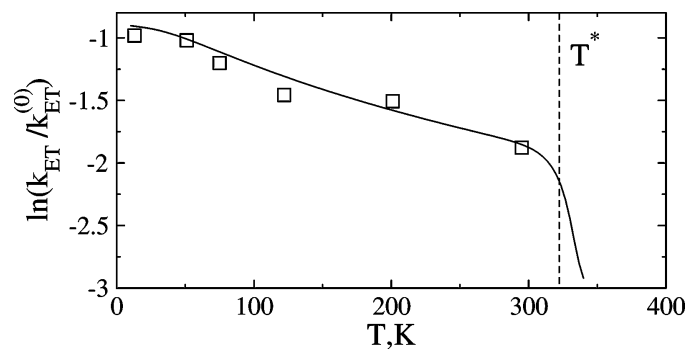

FIGURE 5. Rate of charge separation in $R$. sphaeroides obtained from experiment $t^{34}(\square)$ and from calculations $(-) .{ }^{27}$ The rate is normalized with respect to the non-adiabatic pre-exponent, $k_{\mathrm{ET}}^{(0)}$. The vertical dashed line indicates the approximate position of the turnover temperature, $T^{*}$.

mechanical calculations of the molecular charge distribution of quinoxaline in the initial and final charge-transfer states.

Once the dependence of nuclear solvation on the observation frequency, $\omega_{\text {obs }}$, is established, one can use this information to calculate the reaction rate. For activated transitions, the frequncy cutoff is determined by the rate constant, $\omega_{\mathrm{obs}}=k_{\mathrm{ET}}$. Therefore, the components of the activation barrier affected by nuclear solvation are reduced by the nonergodicity factor given by the ratio of the nonergodic and ergodic reorganization energies. The same scaling applies to the nuclear component of the reaction free energy:

$$
f=\lambda\left(k_{\mathrm{ET}}\right) / \lambda_{\mathrm{s}}, \quad \Delta G_{\mathrm{s}}\left(k_{\mathrm{ET}}\right)=f \Delta G_{\mathrm{s}}
$$

The overall reaction free energy $\Delta G_{0}$ in eq 8 is the sum of $\Delta G_{\mathrm{s}}\left(k_{\mathrm{ET}}\right)$ from eq 12, the gas-phase energy gap, and the free energy of solvation by the electronic degrees of freedom of the solvent. ${ }^{4}$

Since the activation barrier from eqs 8 and 12 depends on the magnitude of the rate constant, the latter should be sought from a self-consistent equation

$$
k_{\mathrm{ET}} \propto \exp \left[-G^{\mathrm{act}}\left(k_{\mathrm{ET}}\right) /\left(k_{\mathrm{B}} T\right)\right]
$$

The solution of this equation results in reaction rates that can potentially change very sharply when a particular nuclear mode freezes in. Each such event is characterized by its turnover temperature, $T^{*}$, and one might anticipate the existence of several such temperatures in media with complex relaxation hierarchies.

The magnitude of the turnover temperature, $T^{*}$, is determined by the underlying solvent dynamics since the kinetic turnover (dynamical arrest) occurs when the relaxation time of a given solvent mode promoting activation crosses the reaction time. For fast reactions, $T^{*}$ may be quite high. Figure 5 illustrates this fact in application to primary charge separation in the photosynthetic bacterial reaction center of Rhodobacter sphaeroids. ${ }^{34}$ Because of the relatively sluggish protein dynamics, modeled by the dynamics of its side chains, and ultrafast rate of charge separation (ca. $0.3 \mathrm{ps}^{-1}$ ), the turnover temperature falls above the normal physiological range of operation of bacterial photosynthesis. ${ }^{27}$ Photosynthetic charge separation thus occurs in the dynamically frozen medium allowing the robust ${ }^{35}$ and temperature-independent operation of the photosynthetic apparatus. The sensitivity of $T^{*}$ to the electron-transfer matrix element imposes the structural limitation such that the edge-to-edge distance between bachteriochlorophyll cofactors should not exceed $\sim 6 \AA$, as is indeed found in bacterial reaction complexes. An increase in the cofactor separation would produce a downward shift of $T^{*}$ with a possibility of termination of photosynthesis at hot operation conditions. The requirement of a weak dependence on temperature also makes the superexchange mechanism of charge transfer less probable and puts emphasis on sequential electron transfer between the cofactors involved in the electron-transfer chain. ${ }^{36}$

In addition to dynamical freezing of nuclear solvation, there are several other features present in bacterial reaction centers that can potentially distinguish these systems from donor-acceptor complexes dissolved in polar solvents normally described within the framework of crossing parabolas of the Marcus-Hush theory. The primary pair of the reaction center is highly polarizable and is placed in a highly anisotropic environment of the hydrophobic protein core generating a substantial electric field. ${ }^{37}$ As we discuss next, the combination of these two factors, large polarizability and strong electric field, may substantially distort the free energy surfaces from their parabolic shape.

\section{Nonparabolic Free Energy Surfaces}

The electron-transfer theories discussed so far are based on the assumption of equal curvatures of two parabolas describing the initial and final charge-transfer states (eq 1). This assumption is well supported by computer simulations, which show that thermal fluctuations of the electrostatic potential produced by a dense polar (dipolar or quadrupolar) solvent are well described by the Gaussian statistics (see discussion in ref 38). In terms of analytical models, a solute-solvent interaction linear in a thermally fluctuating solvent field projects itself into a Gaussian distribution of the donor-acceptor energy gaps in eq 4 . This observation suggests that going beyond the MarcusHush picture of equal-curvature parabolas requires nonlinear coupling of the solute to the solvent field. Several mechanisms of such nonlinear coupling, including strong solute-solvent electrostatic interaction, have been discussed. Here we focus on the self-polarization energy of the solute electronic subsystem, which depends nonlinearly on the solvent polarization and may potentially produce the strongest alteration of the free energy surfaces. ${ }^{38-40}$

Any system of electrons and nuclei immersed in a solvent gains the energy of electronic self-polarization, $E_{i}^{\text {self }}(\mathbf{P})$, which, for polar solvents, can be defined as a function of dipolar polarization P. Once this energy is different in the two electronic states involved in charge transfer, the difference in self-energies, as well as the donor-acceptor energy gap $\Delta E(\mathbf{P})$, are nonlinear functions of $\mathbf{P}$. The Gaussian statistics of polarization fluctua- 
tions of the pure polar solvent is projected in this case into the non-Gaussian statistics of the energy gap $X$. This scheme of producing nonlinearity ${ }^{41}$ still awaits its general solution. Here we discuss the result of using the lowestorder quadratic expansion of $E_{i}^{\text {self }}(\mathbf{P})$ in $\mathbf{P}$. Electronic selfpolarization is then determined by the dipolar polarizability of the solute.

The problem is significantly simplified when the charge distribution of a donor-acceptor complex is approximated by a point dipole, $m_{0 i}(i=1,2)$. It is sufficient then to consider the scalar projection of the electric field on the direction of the dipole instead of considering the vector polarization field $\mathbf{P}$. Since electronic transitions are activated by nuclear thermal fluctuations, only the electric field related to nuclear solvent coordinates is relevant to the discussion of electron transfer. This nuclear electric field is then the sum of the reaction field, $R_{n}$, in response to the charges of the donor-acceptor complex and some macroscopic filed $F$ resulting from a nonuniform distribution of electric charge. This field is normally zero in polar liquids but becomes very significant (ca. $10^{6} \mathrm{~V} / \mathrm{cm}$, ref 37 ) in biopolymers. The overall solute-solvent potential then becomes

$$
U_{0 \mathrm{~s}}^{i}=-m_{0 i}\left(R_{n}+F\right)
$$

The energy of self-polarization of the solute is determined by the dipolar polarizability, $\alpha_{0 i}$, in each charge-transfer state, and one gets

$$
E_{i}^{\text {self }}=-\alpha_{0 i}\left(R_{n}+F\right)^{2} / 2
$$

Once the difference of $U_{0 \mathrm{~s}}^{i}+E_{i}^{\text {self }}$ in the final and initial states is used to define the fluctuating donor-acceptor energy gap, it becomes a bilinear function of the thermally fluctuating field, $R_{n}$, as shown in eq 16 :

$$
X=\text { const }-\Delta m_{0}\left(R_{n}+F\right)-\Delta \alpha_{0}\left(R_{n}+F\right)^{2} / 2
$$

To the extent that quadratic in the field term (the last summand in eq 16 proportional to the polarizability change $\Delta \alpha_{0}$ ) competes energetically with the linear term (the second summand in eq 16 proportional to the dipole moment change $\Delta m_{0}$ ), the statistics of energy gap fluctuations will deviate from Gaussian resulting in nonparabolic free energy surfaces (Figure 1b). For example, $G_{1}(X)$ (within a constant shift) becomes ${ }^{39}$

$$
G_{1}(X)=\kappa\left[\sqrt{\Delta G_{0}+\kappa^{2} \lambda_{1} /(\kappa-1)-X}-\sqrt{\kappa \lambda_{1}}\right]^{2}
$$

Here, the main factor responsible for nonparabolicity is the parameter $\kappa$, which quantifies the difference in equilibrium curvatures of the two free energy surfaces (eq 1)

$$
\kappa=\left(1-\sqrt[3]{\lambda_{1} / \lambda_{2}}\right)^{-1}
$$

where, for simplicity, $\lambda_{1}<\lambda_{2}$ is assumed. In the limit of equal-curvature parabolas, $\lambda_{1} \rightarrow \lambda_{2}$, $G^{\text {act }}=G_{1}(0)$ transforms into the Marcus equation (eq 8).

The free energy surface given by eq 17 is parabolic close to the minimum $X_{01}$ but becomes linear in one of its wings

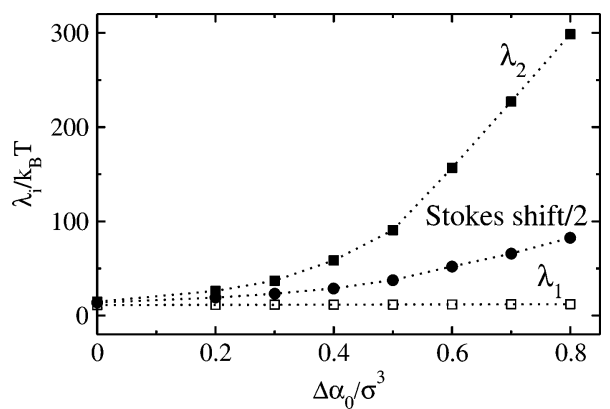

FIGURE 6. Reorganization energies of charge separation $\left(\lambda_{1}\right)$ and charge recombination $\left(\lambda_{2}\right)$ vs the change in the polarizability $\left(\Delta \alpha_{0}\right)$ normalized with the cube of the solvent molecular diameter $(\sigma)$. The points were obtained from Monte Carlo simulations of a polarizable di-atomic solute in dipolar ferroelectric fluid. ${ }^{40}$ Closed circles refer to half of the solvent-induced Stokes shift, which is equal to the solvent reorganization energy in the Marcus-Hush theory but falls between two reorganization energies for nonparabolic free energy surfaces.

far from the minimum. The model thus combines the two paradigms of linear and quadratic free energy surfaces (Figure 1) within one united formalism leading to the energy gap law quadratic close to the top of the bellshaped curve and becoming linear in its exothermic wing (Figure 1c).

The main question regarding the development of these ideas is whether real systems with highly asymmetric reorganization energies can exist. In the absence of wellestablished laboratory evidence, computer simulations suggest that significant asymmetry of the free energy surfaces can be achieved by approximately doubling the solute polarizability by electronic transition. ${ }^{38}$ Macroscopic field $F$ encountered in some anisotropic and inhomogeneous media creates additional mechanisms of reorganization asymmetry. Figure 6 illustrates this by comparing the reorganization energies for charge separation and charge recombination obtained by computer simulation of a polarizable donor-acceptor complex in a model ferroelectric liquid. ${ }^{40}$ The macroscopic electric field present in ferroelectric media creates asymmetry with respect to the flow of electrons in the forward and backward directions resulting in the ratio of corresponding reorganization energies up to a factor of 25 for the system studied in ref 40.

\section{Summary and Perspectives}

It is universally accepted that the activation barrier of chemical reactions in condensed-phase solvents is the free energy barrier composed of enthalpic and entropic components. While the enthalpy component is often available from the Arrhenius analysis, the entropy part is much less understood, primarily because the intercept of the Arrhenius plot reports a combined effect of the rate preexponent and the activation entropy. In application to electrontransfer reactions, the analysis of experimental data has shown a great sensitivity of the results for the rate preexponent to the models used to describe the activation entropy. ${ }^{19}$ As we have stressed in this Account, the reason 
is the fundamentally non-Arrhenius kinetics of the solvent fluctuations occurring on the microscopic length scale and resulting, phenomenologically, in a temperature-dependent activation barrier.

The kinetics converges to the macroscopic Arrhenius limit with increasing the length scale of the interaction potentials involved but never quite reaches it, even for dipolar forces with the longest range of interactions studied so far. It remains to be seen whether studies of the temperature dependence of electron-transfer rates in solvents with an even longer range of interactions (ionic liquids?) will bring a new reference point to this general trend. For the present, the recent combined effort of theory, ${ }^{15,16}$ computer simulations, ${ }^{18}$ and experiment ${ }^{19}$ allows one to hope that the intricate nature of the entropy accumulated in the course of driving the reaction to the top of the activation barrier will ultimately receive a better understanding.

While the studies of the thermodynamics of the solutesolvent interactions are important for establishing the enthalpic and entropic components of the activation barrier, solvent nonergodicity may potentially have more dramatic consequences. The effect of solvent dynamics has traditionally been viewed as a modest one, in the form of modifying the rate pre-exponent (Kramers picture). The nonergodic kinetics change this view, since now the activation barrier, that is, the rate exponent, is affected by the solvent dynamics at the onset of nonergodicity. The fact that the theory and spectroscopic experiment both predict quite sharp changes in the reorganization parameters of electronic transitions offers an opportunity for directed control and switching of redox reactions. Our studies of reactions in supercooled liquids ${ }^{29}$ and liquid crystals $^{42}$ point to a much stronger effect of dynamical nonergodicity on the rate than of any other solvent parameter, thermodynamic state included (isotropicnematic phase transition). It may turn out that this regulation mechanism is widely exploited by natural electron-transfer chains in situations where efficiency and precise tuning are at stake.

The notion of highly asymmetric free energy surfaces, with different reorganization energies for charge separation and charge recombination reactions, brings about new questions regarding the origin of efficiency of natural photosynthesis and directions to optimize artificial photosynthesis. Does the combination of highly polarizable cofactors (primary pair and carotenes in reaction centers) with a strongly inhomogeneous electric field improve the efficiency of natural photosynthesis by creating conditions for unidirectional flow of electrons? Can we improve the efficiency of artificial photosynthesis by combining highly polarizable complexes with anisotropic ferroelectrics and inhomogeneous thin polarized films? At the time of this writing, these are still open questions.

This research was supported by the National Science Foundation (Grant CHE-0616646).

\section{References}

(1) Marcus, R. A.; Sutin, N. Electron transfer in chemistry and biology. Biochim. Biophys. Acta 1985, 811, 265-322.

(2) Barbara, P. F.; Meyer, T. J.; Ratner, M. A. Contemporary issues in electron transfer research. J. Phys. Chem. 1996, 100, 1314813168.

(3) Bixon, M.; Jortner, J. Electron transfer - from isolated molecules to biomolecules. Adv. Chem. Phys. 1999, 106, 35-202.

(4) Newton, M. D. Control of electron transfer kinetics: Models for medium reorganization and donor-acceptor coupling. Adv. Chem. Phys. 1999, 106, 303-375.

(5) Wiederrecht, G. P.; Svec, W. A.; Wasielewski, M. R. Controling the adiabaticity of electron-transfer reactions using nematic liquidcrystal solvents. J. Phys. Chem. B 1999, 103, 1386-1389.

(6) Winkler, J. R.; Gray, H. B. Electron transfer in ruthenium-modified proteins. Chem. Rev. 1992, 92, 369-379.

(7) Davis, W. B.; Ratner, M. A.; Wasielewski, M. R. Conformational gating of long distance electron transfer through wire-like bridges in donor-bridge-acceptor molecules. J. Am. Chem. Soc. 2001, 123, 7877-7886.

(8) Marcus, R. A. On the theory of oxidation-reduction reactions involving electron transfer. I. J. Chem. Phys. 1956, 24, 966-978.

(9) Hush, N. Intervalence-transfer absorption. Part 2. Theoretical considerations and spectroscopic data. Progr. Inorg. Chem. 1967, $8,391-444$.

(10) Kosower, E. M. An Introduction to Physical Organic Chemistry; Wiley: New York, 1968

(11) Hwang, J.-K.; Warshel, A. Microscopic examination of free-energy relationships for electron transfer in polar solvents. J. Am. Chem. Soc. 1987, 109, 715-720.

(12) Tachiya, M. Effect of the dielectric saturation on the rates of electron transfer in polar solvents. Chem. Phys. Lett. 1989, 159, $505-510$.

(13) Reynolds, L.; Gardecki, J. A.; Frankland, S. J. V.; Maroncelli, M. Dipole solvation in nondipolar solvents: Experimental studies of reorganization energies and solvation dynamics. J. Phys. Chem. 1996, 100, 10337-10354.

(14) Raineri, F. O.; Friedman, H. L. Solvent control of electron transfer reactions. Adv. Chem. Phys. 1999, 107, 81-189.

(15) Matyushov, D. V. Solvent reorganization energy of electron transfer in polar solvents. J. Chem. Phys. 2004, 120, 7532-7556.

(16) Milischuk, A. A.; Matyushov, D. V.; Newton, M. D. Activation entropy of electron transfer reactions. Chem. Phys. 2006, 324, $172-194$

(17) Hansen, J. P.; McDonald, I. R. Theory of Simple Liquids; Academic Press: London, 2003.

(18) Ghorai, P. K.; Matyushov, D. V. Solvent reorganization entropy of electron transfer in polar solvents. J. Phys. Chem. A 2006, 110, 8857-8863.

(19) Zimmt, M. B.; Waldeck, D. H. Exposing solvent's role in electron transfer reactions: Tunneling pathway and solvation. J. Phys. Chem. A 2003, 107, 3580-3597.

(20) Milischuk, A. A.; Matyushov, D. V. Quadrupolar solvatochromism: 4-amino-phthalimide in toluene. J. Chem. Phys. 2006, 124, No. 204502 (6 pp).

(21) Matyushov, D. V.; Schmid, R. Optical and radiationless intramolecular electron transitions in nonpolar fluids: Relative effects of induction and dispersion interactions. J. Chem. Phys. 1995, 103, 2034-2049.

(22) Truhlar, D. G.; Garrett, B. G.; Klippenstein, S. J. Current status of the transition-state theory. J. Phys. Chem. 1996, 100, 1277112800.

(23) Weaver, M. J.; McManis, G. E. Dynamical solvent effects on electron-transfer processes: Recent progress and perspectives. Acc. Chem. Res. 1990, 23, 294-300.

(24) Sumi, H.; Marcus, R. A. Dynamical effects in electron transfer reactions. J. Chem. Phys. 1986, 84, 4894-4914.

(25) Walker, G. C.; Aakesson, E.; Johnson, A. E.; Levinger, N. E.; Barbara, P. F. Interplay of solvent motion and vibrational excitation in electron-transfer kinetics: Experiment and theory. J. Phys. Chem. 1992, 96, 3728-3736.

(26) Bagchi, B.; Gayathri, N. Interplay between ultrafast polar solvation and vibrational dynamics in electron transfer reactions: Role of the high-frequency vibrational modes. Adv. Chem. Phys. 1999, $107,1-80$.

(27) Matyushov, D. V. A phenomenological model of electron transfer in solvents near the glass-transition. J. Chem. Phys. 2005, 122, No. 084507 (11 pp).

(28) Hoffman, B. M.; Ratner, M. A. Reorganization energies and rate constants for electron reactions in glass-forming media and proteins. Inorg. Chim. Acta 1996, 243, 233-238. 
(29) Ghorai, P. K.; Matyushov, D. V. Solvent reorganization of electronic transitions in viscous solvents. J. Chem. Phys. 2006, 124, No. 144510 (18 pp).

(30) Jimenez, R.; Fleming, G. R.; Kumar, P. V.; Maroncelli, M. Femtosecond solvation dynamics of water. Nature 1994, 369, 471-473.

(31) Richert, R. Triplet state solvation dynamics: Basics and applications. J. Chem. Phys. 2000, 113, 8404-8429.

(32) Ito, N.; Duvvuri, K.; Matyushov, D. V.; Richert, R. Solvent response and dielectric relaxation in supercooled butyronitrile. J. Chem. Phys. 2006, 125, No. 024504 (8 pp).

(33) Matyushov, D. V. On the microscopic theory of polar solvation dynamics. J. Chem. Phys. 2005, 122, No. 044502 (11 pp).

(34) Fleming, G. R.; Martin, J. L.; Breton, J. Rates of primary electron transfer in photosynthetic reaction centers and their mechanistic implications. Nature 1988, 333, 190-192.

(35) Noy, D.; Moser, C. C.; Dutton, P. L. Design and engineering of photosynthetic light-harvesting and electron transfer using length, time, and energy scales. Biochim. Biophys. Acta 2006, 1757, 90105.

(36) Vos, M. H.; Martin, J.-L. Femtosecond processes in proteins. Biochim. Biophys. Acta 1999, 1411, 1-20.
(37) Steffen, M. A.; Lao, K.; Boxer, S. G. Dielectric asymmetry in the photosynthetic reaction center. Science 1994, 264, 810-816.

(38) Small, D. W.; Matyushov, D. V.; Voth, G. A. The theory of electron transfer: What may be missing? J. Am. Chem. Soc. 2003, 125, 7470-7478.

(39) Matyushov, D. V.; Voth, G. A. Modeling the free energy surfaces of electron transfer in condensed phases. J. Chem. Phys. 2000, $113,5413-5424$

(40) Matyushov, D. V. Reorganization asymmetry of electron transfer in ferroelectric media and principles of artificial photosynthesis. J. Phys. Chem. B 2006, 110, 10095-10104.

(41) Matyushov, D. V.; Newton, M. D. Understanding the optical band shape: Coumarin-153 steady-state spectroscopy. J. Phys. Chem. A 2001, 105, 8516-8532.

(42) Kapko, V.; Matyushov, D. V. Dynamical arrest of electron transfer in liquid crystalline solvents. J. Phys. Chem. B 2006, 110, $13184-13194$

AR7000167 